\title{
Pulmonary hypertension: a clinical case study
}

\begin{abstract}
Pulmonary hypertension can be defined as an elevation of the mean pulmonary arterial pressure greater than $25 \mathrm{mmHg}$ at rest, or more than $30 \mathrm{mmHg}$ while exercising. It is characterized by an increased pulmonary vascular resistance. ${ }^{1}$ The annual mortality rate for pulmonary hypertension is $15 \%$, and there is no cure. ${ }^{2}$ Mortality is due to an increased work load on the right ventricle, which has a relatively thin wall in comparison to the left. Although the right and left ventricles generate the same cardiac output, pulmonary circulation should be significantly less than systemic, but still must be high enough to overcome the forces of gravity and alveolar pressure to perfuse the lung apices in an upright individual. A normal pulmonary artery pressure is a systolic around $25 \mathrm{mmHg}$ over a diastolic around $8 \mathrm{mmHg}$, with a mean pressure of $15 \mathrm{mmHg}{ }^{3}$
\end{abstract}

This report will examine the course of one patient's stay in the Cardiovascular Intensive Care Unit (CVICU) at Southlake Regional Health Centre in Newmarket, Ontario. It will discuss the pathophysiology and clinical manifestations of pulmonary hypertension, the patient's past medical history, course of stay in the CVICU, as well as a summary of how the case was managed.
Volume 2 Issue 6 - 2015

\author{
Samantha Buckle \\ Southlake Regional Health Centre
}

Correspondence: Samantha Buckle (RRT), Southlake Regional Health Centre, Canada, Email sjbuckle2I@hotmail.com

Received: October 13, 2015 | Published: October 21, 2015

\section{Disease process}

\section{Pathophysiology}

The pathophysiology of pulmonary hypertension can vary, depending on the cause. It can be classified as idiopathic/primary, or it can be secondary to a variety of conditions, such as congenital heart defects, collagen vascular diseases, liver cirrhosis, infections, drugs and radiation therapy, and it can occur postoperatively for several reasons. It can be a result of increased pulmonary vascular resistance due to lung conditions such as COPD, or after lung resection surgeries. ${ }^{1}$ Secondary pulmonary arterial hypertension due to cardiac or respiratory diseases can be caused by volume or pressure overload leading to proliferation of pulmonary vasculature and therefore obstruction. Lung parenchymal diseases lead to vascular changes causing vasoconstriction. This increased vascular resistance causes increased right ventricular after load, and eventually hypertrophy and failure. ${ }^{4}$ When lung parenchyma is destroyed (as in COPD), there is a destruction of vasculature as well. Hyperinflation of the lungs compresses vascular beds, thus resulting in an increase in PVR. Polycythemia, which can result from chronic hypoxemia, increases the viscosity of the blood and contributes to hypertension as well, and alveolar hypoxia also causes pulmonary vasoconstriction. Over time, this leads to hypertrophy of the tunica media of the pulmonary vessels, fibrosis of the tunica intima, and an overall narrower lumen. This increase in vascular resistance and pressures leads to an increased right ventricular afterload, leading to dilation and hypertrophy of cardiac muscle mass in order to maintain cardiac output. ${ }^{1}$

Pulmonary hypertension commonly occurs post-operatively, especially after pneumonectomies, lobectomies, and cardiac surgeries, and most frequently after those surgeries that involve repair or replacement of a valve. Chronic left sided heart issues alter the structure and tone of pulmonary vasculature, causing the resultant pulmonary hypertension to be persistent. Factors contributing to the severity of this hypertension include fluid overload, left-ventricular failure, acute lung injury or acute respiratory distress syndrome, pulmonary emboli, and acidosis. Pulmonary hypertension can occur post-operatively due to factors related to the surgery itself. ${ }^{5}$ Any sort of injury to the pulmonary endothelium can alter the balance of vasoactive mediators including thromboxane, prostaglandin, and prostacyclin. For example, if thromboxane and prostacyclin are manufactured out of proportion to each other, the vessels favour constriction, and tone is increased. The endothelium may now release less of the vasodilator nitric oxide (NO) to counteract this effect. Some agents involved in this process, called mitogens, can then cause remodeling of vessel walls and smooth muscle growth in the tunica media. A pulmonary thromboembolism could also occur as a result of surgery, leading to pulmonary hypertension in a similar manner. ${ }^{5}$ Anesthesia may also play a role in increasing pulmonary vascular tone in the post-operative period directly due to its actions on the vasculature, or in an indirect manner through cytokine release. Cytokines, and other inflammatory mediators that may be released, may depress cardiac function, endothelial injury, and modulation of NO or endothelin-1 release, which can increase vascular tone. ${ }^{5}$

\section{Clinical manifestations}

Pulmonary hypertension has few specific symptoms, and when it is secondary it presents as the underlying condition. Other symptoms include dyspnea and possibly syncope on exertion, chest pain, fatigue, lethargy, anorexia, and right upper quadrant pain. Exertional angina sometimes occurs when pulmonary hypertension is secondary to mitral stenosis. ${ }^{4}$ On physical examination, one may hear an increased intensity of the pulmonic component of the second heart sound, and a systolic ejection murmur may be heard. A right ventricular heave may be palpable. Once right ventricular failure develops, severe fluid retention can occur, and jugular venous distension, hepatomegaly, ascites, and peripheral edema may be seen. Other symptoms may include cough, hemoptysis, and occasionally hoarseness due to the 
distended pulmonary artery compressing the recurrent laryngeal nerve. ${ }^{4}$ The term "cor pulmonale" refers to right ventricular failure that occurs as a result of lung disease causing an increased PVR and therefore increased after load. ${ }^{4} \mathrm{~A}$ failing right ventricle is indicated by a high right-atrial pressure, especially when a low cardiac index is also present. The failure of the right ventricle is probably in part due to hypoxia and cardiac ischemia, contributed to by underlying coronary atherosclerosis. A drop in systemic BP below the PAP compromises perfusion to the right ventricle, which could lead to ischemia and cardiovascular collapse. This is known as a pulmonary hypertensive crisis or acute right heart syndrome, and unless prompt measures are taken, patients will become extremely hypotensive, and that, along with hypoxemia and a metabolic acidosis, will lead to death. ${ }^{5}$

\section{Diagnostic procedures}

Diagnosis of pulmonary hypertension involves looking carefully at the patient's history to determine the underlying cause, and the family history to see if there is a genetic link, which is more indicative of idiopathic pulmonary hypertension. There are a variety of tests that can be done to make a definitive diagnosis. Laboratory testing includes a complete blood count and coagulation studies, as well as ABGs. Other lab tests can look for hepatic or infectious causes, as well as collagen-vascular diseases. ${ }^{4}$ Chest radiography may show an enlarged heart, especially on the right side, and may give a clue as to an underlying lung disease, such as COPD or interstitial lung diseases. Results from this may lead to a chest CT or spiral CT being necessary to take a better look at any abnormalities, and to help rule out a thromboembolic cause. Two dimensional echocardiography is a very common test that is very valuable in diagnosis of this disease, and it shows any valvular abnormalities (tricuspid regurgitation is common with pulmonary hypertension) and dilation or thickness of the right ventricle. Doppler echocardiography can be used to estimate PAP using the modified Bernouli Equation. Ventilationperfusion scanning of the lungs may be done to determine if the cause is thromboembolic, and depending on the results performance of pulmonary angiography may be necessitated. ${ }^{4}$ Pulmonary function testing will show a decreased diffusing capacity (DLCO) in all patients with pulmonary hypertension, and spirometry results showing an obstructive and/or restrictive pattern can help point to the cause. Right-sided cardiac catheterization is the most definitive test for diagnosis and quantification. It can exclude left-heart dysfunction and intra-cardiac shunt, and be used to measure cardiac output. It can provide measurements of PVR and be used to assess responsiveness to vasodilators. An electrocardiogram can show right ventricle hypertrophy by showing right-axis deviation and possibly a right bundle-branch block. ${ }^{4}$

\section{Treatment}

There is no cure for pulmonary hypertension, but the goal of treatment is to take the work off of the relatively thin-walled right ventricle. If secondary, management of pulmonary hypertension involves treating the cause, if possible. For example, if the cause is deemed to be pulmonary embolism, specific treatments for that exist. If the hypertension is mild to moderate and the patient is clinically stable, still has good right ventricular function, then once fluid status and systemic blood pressure are optimized only monitoring is required. Conversely, acute right heart syndrome is an emergency and active treatment is required. ${ }^{5}$ No matter what the cause, fluid balance is important. The Frank-Starling curve should be optimized for best results on ventricular function and cardiac output, and fluid challenges can be performed to assess this ventricular function, as well as kidney function. Too much fluid can over-fill an already dilated right ventricle which can impair left ventricular filling as well. On the other hand, under-hydration can lead to systemic hypotension, which can lead to acute right heart syndrome. Studies have shown that tidal volumes $>6$ millilitres/kilogram and plateau pressures $>30 \mathrm{cmH}_{2} \mathrm{O}$ can add to right heart dysfunction. ${ }^{5}$ If ventilator settings and fluid volume are optimized but systemic hypotension continues, vasopressors should be initiated to maintain systemic above pulmonary pressures, but too many presssors can increase myocardial oxygen consumption and right-ventricular afterload. No ideal vasopressor has been found for this situation, but some studies recommend vasopressin because it can also dilate pulmonary arteries. ${ }^{5}$ Inotropes may also be used to restore cardiac output and improve systemic perfusion by increasing cardiac contractility, but because they vasodilate systemically, they often must be used in combination with pressors. Phosphodiesterase-3 inhibitors such as amrinone and milrinone tend to be the preferred inotropes for this situation because they tend to augment stroke volume more without the heart rate increase caused by catecholamines. ${ }^{5}$

The use of pulmonary vasodilating agents should be attempted after everything else is stabilized. The main goal is to reduce rightventricular after load in order to improve performance. This is sometimes difficult to accomplish because some patients have a fixed high pulmonary vascular tone that has little capacity to relax. Also, many of the agents employed to dilate pulmonary vasculature also dilate systemic vasculature, worsening hemodynamic instability and oxygenation. Some examples are intravenous nitroglycerin and nitroprusside, calcium channel blockers, angiotensin-convertingenzyme inhibitors, and hydralazine. ${ }^{5}$ Intravenous prostacyclins such as epoprostenol and treprostinil are frequently used as rescue therapies for severe pulmonary hypertension due to their potent vasodilatory effects. Of these, epoprostenol is preferred because it has a much shorter half-life, and is thus safer for unstable patients. They have been proven to lower PAP more than nitroglycerin, however, when given intravenously they both lack specificity to the pulmonary system and can therefore worsen systemic hypotension. Inhaled prostacyclins are now growing in popularity due to a much greater pulmonary specificity, and also because they increase blood flow to ventilated areas of the lungs, improving the ventilation-perfusion ratio and oxygenation. Use of inhaled epoprostenol has been growing for acute right heart syndrome. It is continuously nebulized through a ventilator circuit or via face mask, and one study ${ }^{5}$ showed a decrease in mean PAP by more than $10 \mathrm{mmHg}$, as well as improved cardiac output with no change in systemic BP, when given following cardiac and lung surgeries. However, suitability for long-term administration has not yet been established. ${ }^{5}$

Endothelin receptor antagonists such as bosentan and ambrisentan are used therapeutically in the long-term for pulmonary hypertension, and are considered favourable for chronic heart failure. That being said, they have not been studied for acute pulmonary hypertension and tend to cause fluid retention. ${ }^{5} \mathrm{NO}$ causes vasodilation and discourages smooth muscle cell proliferation. It offers the benefits of potent pulmonary vasodilation, little systemic effects, and improving oxygenation. It is delivered with a commercial delivery device and a ventilator circuit or a face mask. It is one of the most wellknown drugs used in the treatment of pulmonary hypertension, but several randomized trials found no appreciable results on mortality or hemodynamics, and two thirds developed a withdrawal syndrome with systemic hypotension and poor oxygenation. ${ }^{5}$ 
Anticoagulants have proven to be of benefit in pulmonary hypertension that has developed atrial fibrillation as a result of the heart failure in order to prevent venous thromboembolism. ${ }^{4}$ Calcium channel blockers also seem to be of greater benefit in idiopathic than secondary forms, and only after the patient has a proven response to vasodilators. Calcium channel blockers work by not allowing calcium to enter voltage-gated channels of vascular smooth muscle. ${ }^{4}$ Phospodiesterase-5 enzyme inhibitors such as sildenafil may be a beneficial long-term treatment for pulmonary hypertension. Sildenafil works by promoting smooth muscle relaxation in lung vasculature, decreasing PAP and increasing $\mathrm{CO}$. Cardiac glycosides such as digoxin are used to prevent and treat some supraventricular arrhythmias that may be associated with pulmonary hypertension, and for those patients that also have left (but not right) sided heart failure. ${ }^{4}$ Diuretics, especially loop diuretics like furosemide, are used for patients that have developed cor pulmonale and as a result have developed hepatomegaly and dependant edema. However, these diuretics may lower systemic blood pressure too much and other drugs are often required to prevent that. ${ }^{4}$

Supplemental oxygen is a common therapy for pulmonary hypertension. As mentioned previously, alveolar hypoxia causes pulmonary vasoconstriction, so oxygen saturations should be kept greater than $90 \%$. Surgery may be an option for some patients with pulmonary hypertension. Atrial septostomy is a procedure that uses a balloon to create a hole in the atrial septum to allow right to left shunting, increasing systemic perfusion and taking pressure off of the right heart, but with a fall in arterial oxygen levels. ${ }^{1}$ Single or double lung transplant or heart-lung transplant is a successful option in some patients with severe cardiac impairment and who do not respond to pulmonary vasodilators. This causes an immediate improvement in PAP and heart function after surgery, but requires lifelong immunosuppression and there is an increased mortality over time. ${ }^{1}$

\section{Patient information}

The patient is an 83year old with, according to the medical chart, a past medical history including hypothyroidism, chronic atrial fibrillation, chronic anemia, and $\mathrm{T}_{2} \mathrm{~N}_{0}$ lung cancer requiring lobectomy of the right middle and right lower lobes 19years prior to admission. The patient had a 60 pack year smoking history but quit, and had a myocardial infarction 15 years earlier. Over the few months prior to admission to Southlake, the patient complained of shortness of breath on exertion and mild orthopnea. The pre-admission diagnosis was treated coronary artery disease and aortic aneurysm. It was discovered there was calcified aortic stenosis and calcification of the mitral valve annulus with no stenosis. An echocardiogram revealed an estimated pulmonary artery systolic pressure of $64 \mathrm{mmHg}$, a dilated aorta and enlarged left atrium. A chest X-ray done at Southlake one month before admission showed overinflation and coarse markings indicative of chronic obstructive pulmonary disease (COPD), along with marked pleural thickening on the right side.

The patient was admitted to Southlake to have an aortocoronary bypass times one, tissue aortic valve replacement, and replacement of the ascending aorta. Under anesthesia during the surgery, the pulmonary artery pressure (PAP) measured via a pulmonary artery catheter was $55 \mathrm{mmHg}$, with a suspicion that it would be higher while the patient was awake. There were initially no complications from the surgery. After the operation, the patient was brought to the CVICU and was received intubated with a size 7.5 endotracheal tube and was fully ventilated, as is the norm for patients after cardiac surgery at this hospital. There was a Swan-Ganz catheter in situ. The patient presented with a heart rate of 79 beats per minute, blood pressure of $120 / 47 \mathrm{mmHg}$, PAP of $42 / 15 \mathrm{mmHg}$, and central venous pressure (CVP) of $6 \mathrm{mmHg}$. Cardiac output was 4.2 litres per minute (LPM), systemic vascular resistance (SVR) was 1180 dynes* $\mathrm{s} / \mathrm{cm}^{5}$ and pulmonary vascular resistance (PVR) was 171 dynes*s $/ \mathrm{cm}^{5}$. The highest PAP noted that day in CVICU was $49 / 23$ with a blood pressure (BP) at that time reading 101/44 via an arterial line. On auscultation, air entry was diminished to the right side and breath sounds were described as fair.

\section{Clinical course \\ CVICU day I}

The patient was received in the CVICU after cardiac surgery already intubated and was set up on the Puritan Bennett 840 ventilator in the assist/control volume control mode with a set respiratory rate (RR) of 14 breaths per minute (bpm), tidal volume of 450 millilitres, flow of 55litres per minute, positive end- expiratory pressure (PEEP) of $5 \mathrm{cmH}_{2} \mathrm{O}$, flow sensitivity of 3 litres per minute, and fractional inspired oxygen $\left(\mathrm{FiO}_{2}\right)$ of 0.50 . Humidity was provided via a heat and moisture exchanger (HME). There was a plan to wake, wean, and extubate. Later that evening after beginning to wake up, the patient was put in a spontaneous mode with a pressure support of $12 \mathrm{cmH}_{2} 0$, PEEP of $5 \mathrm{cmH}_{2} \mathrm{O}$, and $\mathrm{FiO}_{2}$ of 0.40 .

\section{CVICU day 2}

In the early morning hours of the following day, the patient was weaned to a pressure support of $6 \mathrm{cmH}_{2} \mathrm{O}$ with all other settings remaining the same. An arterial blood gas taken at this time was $\mathrm{pH}$ 7.29, $\mathrm{PaCO}_{2} 45, \mathrm{PaO}_{2} 178, \mathrm{HCO}_{3}-21.6, \mathrm{SaO}_{2} 99 \%$. The anesthetist was aware of the acidemia, but said to go ahead with the extubation. The patient was extubated with no issues initially. The PAP around the time of extubation was 52/23, with a BP of $106 / 47$ and CVP of 25. Later that day, urine output decreased so subsequently the patient was given 2litres of fluid and the dose of norepinephrine(Levophed) was increased. Overnight PAP (systolic) rose to around 60 to $70 \mathrm{mmHg}$, and then later up to 85 to $90 \mathrm{mmHg}$, with a central venous pressure of $30 \mathrm{mmHg}$, and $\mathrm{SpO}_{2}$ was steadily declining. A decision was made to re-intubate the patient.

\section{CVICU day 3}

Shortly after midnight on that night, the patient was taken back to the operating room (OR). An echocardiogram performed could not rule out cardiac tamponade. Once in the OR, it was discovered that there was no tamponade present, but there was very poor right ventricular function. The patient was then returned to the CVICU and placed on pressure control ventilation with a $\mathrm{RR}$ of 18 breaths per minute, pressure control of $14 \mathrm{cmH}_{2} \mathrm{O}, \mathrm{PEEP}$ of $5 \mathrm{cmH}_{2} \mathrm{O}$, and $\mathrm{FiO}_{2}$ of 0.8 . Inhaled epoprostenol (Flolan) was initiated at this time at a dose of 20 000units via a nebulizer through a heated humidity ventilator circuit. The nebulizer was kept at a volume of 15 millilitres with a pump set at 8 millilitres per hour. A dialysis catheter was inserted and sustained low-efficiency dialysis (SLED) was begun to help improve her hemodynamic stability and metabolic acidosis. SLED was chosen over other types of hemodialysis or renal replacement therapy because of the improved hemodynamic stability associated with it. 1.5 litres of fluid was removed with the dialysis on the first day. The patient was not triggering breaths above the set rate at this point. Later that 
morning $\mathrm{FiO}_{2}$ was decreased to 0.60 , RR was increased to 26 breaths per minute and pressure control increased to 16 due to an $\mathrm{ABG}$ showing $\mathrm{pH} 7.21 / \mathrm{PaCO}_{2} 47 / \mathrm{PaO}_{2} 213 / \mathrm{HCO}_{3}-18.8 /$ Base excess (BE) $-8.6 / \mathrm{SaO}_{2} 100 \%$. An expiratory pause maneuver performed several hours later indicated airtrapping with a total PEEP of $11 \mathrm{cmH}_{2} \mathrm{O}$, probably in part due to the two expiratory filters required while Flolan is in line, and as a result a respiratory therapist (RT) decreased the set $\mathrm{RR}$ to 22 breaths per minute. Breath sounds at this point were described as coarse crackles.

\section{CVICU day 4}

The patient's ventilator settings were not adjusted much over the next several days, except for a decrease in $\mathrm{FiO}_{2}$ to 0.30 . Around 1300 on this day, the Flolan dose was weaned to 10000 units with all other parameters remaining the same. At this time PAP was 36/20. By 1830 that same day PAP was steadily rising and increased to $82 / 22$. As a result, the dose of Flolan was returned to 20000 units. Dialysis removed 2.5litres of fluid on day 4 .

\section{CVICU day 5}

The next morning the patient was trialed on a pressure support of $12 \mathrm{cmH}_{2} \mathrm{O}$, PEEP of $5 \mathrm{cmH}_{2} \mathrm{O}$, and $\mathrm{FiO}_{2}$ of 0.30 .15 minutes later the patient had become tachypneic with small tidal volumes and was returned to pressure control ventilation with a set RR of 22, pressure control of 16, PEEP of 5, and $\mathrm{FiO}_{2}$ of 0.30 . Within several hours the RT again tried the patient on a pressure support of 12. After settling on this mode, an ABG showed $\mathrm{pH} 7.21 / \mathrm{PaCO}_{2} 55 / \mathrm{PaO}_{2}$ 195/ $\mathrm{HCO}_{3}-$ $22 / \mathrm{BE}-5.6 / \mathrm{SaO}_{2} 100 \%$. The RT then returned the patient to pressure control to improve the respiratory acidosis, this time with a set RR of 20 and pressure control of 14. During the afternoon PAP rose to $91 / 24$ with a BP of $125 / 42$. PVR was 307 and SVR was 658 . A push of nitroglycerin was given and the patient was put in a reverse Trendelenburg position. This seemed to help resolve the pulmonary hypertensive episode and within several hours PAP stabilized around 46/24.A transthoracic echocardiogram performed on day 5 revealed trivial aortic regurgitation and moderate mitral, tricuspid, and pulmonic valve regurgitation. The left atrium was dilated with a normal sized left ventricle and grade II left ventricular function. The right atrium and ventricle were both enlarged, and severe pulmonary hypertension could be seen. 3.5litres of fluid was removed with dialysis.

\section{CVICU day 6}

The patient spent most of day 6 with a set RR of 20 and a pressure control of 18. At 0800, the Flolan dose was decreased to 10 000units once again. With PAP remaining stable, at 1030 the dose was decreased to 5000 units. At 1400 the dose was halved one more time to 2500 . Two hours later the patient was taken off Flolan completely and switched to a pressure support mode. The next $\mathrm{ABG}$ drawn was $\mathrm{pH}$ 7.38/ $\mathrm{PaCO}_{2} 37 / \mathrm{PaO}_{2} 84 / \mathrm{HCO}_{3}-21.9 / \mathrm{BE}-2.9 / \mathrm{SaO}_{2} 96 \%$. Throughout most of the day systolic PAP remained around $60 \mathrm{mmHg}$ despite the Flolan changes, but after the Flolan was turned off, the patient was turned onto their left side and the systolic pressure rose to greater than $70 \mathrm{mmHg}$. Returning to their right side two hours later brought PAP down to $59 / 22$.

\section{CVICU day 7-8}

The morning of day 7 the patient was weaned from a pressure support of 12 to a pressure support of 6 , still with a PEEP of 5 and $30 \% \mathrm{FiO}_{2}$. All $\mathrm{ABG}$ done this day remained stable, with all values in the accepted normal ranges. A sputum culture and sensitivity test was performed and the results were negative. The next morning the patient was having an episode of desaturation requiring a $\mathrm{FiO}_{2}$ increase to 0.60 and a PEEP of 7.5. The patient had the final course of dialysis this day, and possible extubation was discussed. However, the patient was demonstrating accessory muscle use on exhalation and as a result was returned to a pressure control mode which resolved the increased work of breathing.

\section{CVICU day 9}

On day 9 the patient had a computed tomography (CT) scan of the head performed. A meningioma was found on the left side of the patient's brain, which was deemed to be benign. Meningiomas are most commonly found in elderly woman, and are often asymptomatic, with treatment not always necessary. ${ }^{7}$ Following the scan, the patient was once again returned to a pressure support of 12, with a PEEP of 7.5 and $\mathrm{FiO}_{2}$ of 0.35 . A spontaneous breathing trial was initiated with a pressure support of 6 on a PEEP of 5, and $\mathrm{FiO}_{2}$ 0.35. The patient passed the spontaneous breathing trial. $\mathrm{An} \mathrm{ABG}$ drawn during this trial was $\mathrm{pH} 7.35 / \mathrm{PaCO}_{2} 48 / \mathrm{PaO}_{2} 91 / \mathrm{HCO}_{3}-26.5 / \mathrm{BE} 0.6 / \mathrm{SaO}_{2} 97 \%$. A cough and gag reflex and a cuff leak were present, but the patient was not consistently following commands. Despite that, the patient was extubated to $40 \%$ oxygen via large volume nebulizer. There were initially no complications with the extubation, but the patient was only sporadically responsive. An ABG was done several hours later and it showed $\mathrm{pH} 7.32 / \mathrm{PaCO}_{2} 51 / \mathrm{PaO}_{2} 157 / \mathrm{HCO}_{3}-26.3 / \mathrm{BE}-0.1 / \mathrm{SaO}_{2} 99 \%$.

\section{CVICU day 10}

Throughout the rest of the day after the extubation and overnight, the patient became progressively more acidemic. The following morning an $\mathrm{ABG}$ showed $\mathrm{pH} 7.22 / \mathrm{PaCO}_{2} 57 / \mathrm{PaO}_{2} 96 / \mathrm{HCO}_{3}-23.3 /$ $\mathrm{BE}-4.5 / \mathrm{SaO}_{2} 96 \%$. The decision was made to re-intubate. It was a reportedly awkward intubation with a size 7.5 endotracheal tube. A bronchoscopy was performed and the washings were normal. The patient was placed in pressure control ventilation with a pressure control of 14 , rate of $20, \mathrm{FiO}_{2}$ of 0.40 , and PEEP of 12. Later that day a bedside tracheostomy was attempted for difficulty to wean, but there was profuse bleeding and it was aborted. The patient was then taken to the OR where a size $6.0 \mathrm{~mm}$ inner diameter cuffed, fenestrated Shiley tracheostomy tube was inserted.

\section{CVICU day I I}

The following morning the patient was put on a pressure support of 12 , with a PEEP of 8 , and $\mathrm{FiO}_{2}$ of 0.30 and was breathing at a RR of 22 and getting tidal volumes around 350millilitres. An ABG taken after settling on this mode was $\mathrm{pH} 7.42 / \mathrm{PaCO}_{2} 39 / \mathrm{PaO}_{2} 112 /$ $\mathrm{HCO}_{3}-25.3 / \mathrm{BE} 0.8 / \mathrm{SaO}_{2} 98 \%$. The patient was then trialed off of the ventilator with a trach mask and large volume nebulizer with the $\mathrm{FiO}_{2}$ set at 0.40 . An ABG was drawn after about one hour on the trach mask trial and showed $\mathrm{pH} 7.28 / \mathrm{PaCO}_{2} 54 / \mathrm{PaO}_{2} 203 / \mathrm{HCO}_{3}$ - 25.4/BE$1.7 / \mathrm{SaO}_{2} 100 \%$. The patient had become lethargic, hypotensive, and tachycardic, and as a result was returned to the ventilator.

\section{CVICU days I2-22}

Over the next week trach mask trials were performed every day. Most days the patient did two or three trials a day, lasting several hours each before becoming tired with increased work of breathing and climbing $\mathrm{PaCO}_{2}$ and as a result was rested on pressure support in between trials and overnight. 


\section{CVICU day 23-24}

On the $23^{\text {rd }}$ day in the CVICU the patient became more acidemic, with a $\mathrm{pH}$ of 7.28 and a $\mathrm{PaCO}_{2}$ of $66 \mathrm{mmHg}$, and was therefore put in pressure control mode with a set RR of 20 , pressure control of 18 , inspiratory time of 0.95 seconds, PEEP of $5 \mathrm{cmH}_{2} \mathrm{O}$, and $\mathrm{FiO}_{2}$ of 0.40 . The patient was receiving a tidal volume of around 480 millilitres. The $\mathrm{RR}$ was later increased to $24 \mathrm{bpm}$ due to a $\mathrm{pH}$ of 7.26 and $\mathrm{PaCO}_{2}$ of 73. Before too much longer oxygen requirements increased requiring a $\mathrm{FiO}_{2}$ of 0.50 and a PEEP of 7.5, and the pressure control was lowered to 16. Several hours later the patient was trialed on a pressure support of 10, with the PEEP and $\mathrm{FiO}_{2}$ remaining the same. Within three hours the patient was returned to pressure control because of worsening ABGs. The set RR was 16 with a pressure control of 12 . Around 1500 that day PAP was $84 / 29$, with a PVR of 300 and SVR of 683 . Inhaled Flolan was started again at a dose of 20000 units. A bronchoscopy was performed and a large amount of bloody secretions were retrieved. In the sputum culture, pus cells were seen and microbiology indicated moderate growth of enterobacter cloacae complex resistant to all penicillins, cephalosporins, and beta lactamase inhibitors. The patient was diagnosed with pneumonia and was prescribed imipenem. The patient was deemed to be in congestive heart failure (CHF) with resultant pulmonary edema, and to be in renal dysfunction due to a decreased urine output with the inability to diurese, despite being on furosemide (Lasix). Pulmonary infiltrates seen on chest X-ray were worsening. Level of consciousness was continually decreasing and the patient was becoming more difficult to awaken than they had been previously. The patient was given a transfusion of one unit of packed red blood cells due to low hemoglobin. On day 24, set RR and pressure control were both increased to 18 , with a PEEP of 10 and $50 \% \mathrm{FiO}_{2}$. Overnight the patient began to have seizures, with four witnessed seizures that night beginning with twitching in the right arm, then moving to the left arm and right leg. PAP increased with any kind of stimulation.

\section{CVICU days 25-3 I}

The patient was becoming asynchronous with the ventilator by "breath-stacking." As per doctor's orders, an RT switched the patient to a volume control mode with the hopes of the patient being more comfortable. The set tidal volume was 380 millilitres and the set RR was 30. This did increase the minute ventilation which helped resolve the respiratory acidosis. Fentanyl was also ordered to be given when the patient was not coordinating with the ventilator. At 0800 PAP was $76 / 28$, blood pressure was $110 / 46$, and SVR was 387 . By 1400 , PAP was $78 / 24$, BP was $78 / 22$, and SVR was 389 . Milrinone was turned off to increase the SVR and BP. SLED was started once again to help overcome the fluid overload and hopefully decrease the pulmonary edema. 1.9litres of fluid was removed. The tidal volume was later decreased to 350millilitres. The patient's family decided on a do not resuscitate order specifying no compressions. The patient had more seizures on the $26^{\text {th }}$ day, and when the inner cannula of the tracheostomy tube was changed there was a bradycardic episode. Overnight Flolan was weaned to 10000 units, and several hours later it was decreased to 5000 units. On the morning of day 27 Flolan was discontinued. Within two hours systolic PAP was over $100 \mathrm{mmHg}$, and 10 000units of Flolan was started again. The set respiratory rate was increased to $32 \mathrm{bpm}$.

On day 28 an RT tried to return the patient to pressure control, which apparently was not tolerated and as a result, was put in a pressure regulated volume control (PRVC) mode, known as $\mathrm{VC}+$ on the 840 ventilator, which provided adequate volumes with a lower mean airway pressure. The initial settings were a RR of $26 \mathrm{bpm}$, set tidal volume of 350 millilitres, PEEP of $5 \mathrm{cmH}_{2} \mathrm{O}$, and $60 \% \mathrm{FiO}_{2}$. A peak inspiratory pressure of around $40 \mathrm{cmH}_{2} \mathrm{O}$ was required to reach this volume, which slightly decreased once becoming more settled on the mode. The higher pressures required to maintain tidal volume indicate a decreasing lung compliance or increasing airway resistance. The volume later had to be increased to 380 millilitres due to rising $\mathrm{PaCO}_{2}$. An electroencephalogram was performed on this day in question of the patient's neurological status. Dialysis was stopped. During the night of the $28^{\text {th }}$ day, the patient was started on sildenafil (Viagra) to treat the pulmonary hypertension. By morning, the Flolan was weaned to 5000 units. On day 29, it was decided to discontinue the Flolan. This did cause a decrease in mean airway pressure, but systolic PAP rose from around 65 to between 75 and $85 \mathrm{mmHg}$. The following day the patient began having issues with oxygenation requiring an increase in $\mathrm{FiO}_{2}$ to 0.7 and a PEEP of $8 \mathrm{cmH}_{2} \mathrm{O}$. On the $30^{\text {th }}$ day, an RT tried the patient in proportional assist ventilation $(\mathrm{PAV}+$ on the 840$)$ with a percent support of $80 \%$ in an attempt to wean. Unfortunately, the patient became restless with increased work of breathing and lower oxygen saturations and was returned to previous settings, remaining there throughout the following day.

\section{CVICU days 32-35}

On day 32 of the patient's stay, Southlake's PAV weaning protocol was tried once again, where the patient was rested on $80 \%$ support with several short periods on $35 \%$ support. ${ }^{6}$ The next day, $\mathrm{PaCO}_{2}$ rose to $62 \mathrm{mmHg}$, so the patient was placed on a pressure control of $18, \mathrm{RR}$ of 24, PEEP of 10, and $\mathrm{FiO}_{2}$ of 0.50 . On day 34 the PAV protocol was continued. On day 35 the respiratory acidosis worsened once again and the patient was returned to pressure control ventilation. Chest $\mathrm{X}$-rays were now showing an interstitial lung disease (ILD) pattern, but what caused that is unknown. A sputum culture was negative. The patient was no longer having seizures at this point, but was still not responsive despite being on no sedation.

\section{CVICU days 36-37}

The patient was returned to PAV+ on the morning of day 36, but within six hours was put in pressure support ventilation due to increased work of breathing, decreased $\mathrm{SpO}_{2}$, and tidal volumes less than 300 millilitres. Two hours later volumes were once again less than 300 , respiratory rate was over 40 breaths per minute, and the patient was working to breathe, and was therefore put in pressure control again. Despite these changes, the patient remained in a respiratory acidosis. Salbutamol (Ventolin) and ipratropium bromide (Atrovent) were ordered.On day 37 the patient was put on PAV $+80 \%$ again, and later was put down to the $35 \%$ level for 30 minutes as per the protocol, but it was not tolerated. The patient was having frequent premature ventricular contractions (PVCs). Several hours later the patient was becoming tired, and was put in pressure control due to $\mathrm{pH} 7.25 / \mathrm{PaCO}_{2}$ $64 / \mathrm{PaO}_{2} 121 / \mathrm{HCO}_{3}-28.1 / \mathrm{SaO}_{2} 98 \%$. The patient continued to have bloody secretions.

\section{CVICU Days 38-40}

Over the next several days, the set RR was gradually increased to 32 and pressure control was increased to 22 due to a worsening acidosis. The pressure control was later increased to $25 \mathrm{cmH}_{2} \mathrm{O}$ due to a $\mathrm{pH}$ of 7.15. Oxygen requirements were progressively increasing as well, at one point requiring an $\mathrm{FiO}_{2}$ of 1.0 , but that was eventually weaned to 0.6 . The chest X-ray was now showing a left upper lobe atelectasis which looked like a new pneumonia, so the patient was 
started on vancomycin. On the night of day 39, the patient's atrial fibrillation converted into a ventricular tachycardia. An amiodarone infusion was quickly begun and the arrhythmia was resolved, and blood pressure eventually stabilized. On the $40^{\text {th }}$ day of the patient's stay, family decided to withdraw support and the patient passed away.

\section{Laboratory findings}

Once lab results stabilized after the heart surgery, they remained fairly consistent. That is, until day 22 of the patient's stay when leukocytes rose from 10.4 to $16.5 \times 10^{9} / \mathrm{L}$. Within the differential, lymphocytes increased the most at this time from 8.4 to $14.9 \times 10^{9} / \mathrm{L}$, which is consistent with a chronic bacterial infection. The patient had a low anion gap throughout the time in hospital. Hemoglobin levels remained in the $80-100 \mathrm{~g} / \mathrm{L}$ range which goes along with the history of chronic anemia, and as a result received several transfusions of packed red blood cells over the time in the CVICU. Albumin levels were also low, and the patient received albumin treatments. This is consistent with renal dysfunction. On day 25 when dialysis was initiated again, serum creatinine was $107 \mu \mathrm{mol} / \mathrm{L}$, and blood urea nitrogen was $30.4 \mathrm{mmol} / \mathrm{L}$. These are elevated values, which indicates that kidney function is declining. ${ }^{8}$

\section{Conclusion}

\section{Summary and Critique of Case Management}

The patient came to Southlake hospital for an aortocoronary bypass, aortic valve and ascending aorta replacement and after the surgery, was admitted to the CVICU, taken off sedation, weaned, and extubated, as per hospital standards after cardiac surgery. That night, pulmonary artery pressures were increasing and approaching systemic blood pressure. The patient was re-intubated and taken back to the OR with suspected tamponade, but nothing was found. The patient was then returned to the CVICU where inhaled Flolan (epoprostenol) and dialysis were initiated to deal with the pulmonary hypertension, metabolic acidosis, and issues with fluid balance. Over the next week Flolan and ventilation were weaned and the patient was then extubated, only to be re-intubated due to worsening ABGs. A tracheostomy tube was then inserted for prolonged ventilation and difficulty with weaning, and then trach mask trials were performed daily for short periods of time as the patient easily tired. Trials eventually were put on hold and Flolan was initiated once again due to rising PAP. Secretions suctioned through the tracheostomy tube were becoming bloody, and the patient was diagnosed with pneumonia, $\mathrm{CHF}$, and renal dysfunction. The patient was having episodes of acute right heart syndrome developing systemic hypotension with PAP almost equalling blood pressure, which can be deadly if not promptly treated. ${ }^{5}$

The previous right-sided bilobectomy was probably a big part of the hypertensive issues experienced during the patient's stay at Southlake because this loss of lung tissue corresponds to a loss of lung volume, which would cause an increase in PVR, as well as possibly having COPD. The risk of developing pulmonary hypertension following a right-sided lung resection is greater than with a leftsided one. ${ }^{9}$ The patient had an elevated baseline PAP to begin with, and the major cardiac surgery likely aggravated the underlying condition. The pulmonary hypertension could also be attributed, in part, to left heart obstruction. The patient had issues with all of the heart valves, and the mitral valve regurgitation could cause a backup of blood into the pulmonary veins.. That being said, pulmonary capillary wedge pressures (PCWP) remained relatively normal, which points to the pulmonary hypertension being more of a result of a lung problem then cardiac issues. The right ventricular function continued to worsen throughout the patient's stay, despite the best efforts of the interprofessional team to keep hemodynamics stable. The hospital stay was complicated by a possible ventilator associated pneumonia, as well as renal dysfunction and pulmonary edema, which are being attributed to the pulmonary hypertension, and could also be a result of valvular insufficiencies and fluid overload. A possible adverse effect of Flolan is pulmonary edema as a result of increased forward flow from the right ventricle. ${ }^{10}$ The seizures that the patient began having later in the course of the stay are suspected to be a result of dialysis dysequilibrium syndrome, a rare complication of dialysis caused by urea gradients and resulting in cerebral edema and neurological symptoms, potentially leading to coma or death. ${ }^{11}$ The pathophysiology of this is beyond the scope of this paper.

This patient was a difficult wean from the ventilator, and as such required a tracheostomy. The patient was tried on pressure support frequently by respiratory therapists, and after receiving the tracheostomy was tried off of the ventilator everyday as long as the patient was stable. Studies have shown that tidal volumes less than 6millilitres/kilogram should be used for patients with pulmonary hypertension, ${ }^{5}$ so at times this patient was likely receiving volumes too large for the condition, especially for someone who is missing most of the right lung. Having said that, the patient often had issues with hypercapnia requiring an increase in respiratory rate and tidal volume in order to maintain a minute ventilation high enough to normalize $\mathrm{pH}$. Inhaled epoprostenol and nitric oxide are two of the most common pulmonary vasodilators used for pulmonary hypertension. Studies disagree on which of the two is the best option, but as NO was not available at this hospital and it is more expensive, Flolan is a good choice that seemed to work for this patient. According to Southlake's policies and procedures regarding the use of inhaled epoprostenal for acute right heart failure, post-operative pulmonary hyptertension, and as a rescue treatment for acute respiratory distress syndrome (ARDS), a positive response is considered to be a decrease in PAP by $15 \%$, which did occur with this patient. ${ }^{10}$ The patient also received other medications such as milrinone, nitroglycerin, and sildenafil for treatment that go along with the current recommendations for pulmonary hypertension.

Pulmonary hypertension is a disease that currently has no cure and is challenging to diagnose and treat. The incidence and prevalence are not completely known as many people go undiagnosed. Much has been learned about the pathophysiology in recent years, but much is still unknown. Further research is necessary into treatment options: comparing and contrasting current ones to determine superiority of one over the other for certain situations, as well as into new treatment options and finding a cure. ${ }^{5}$

\section{Acknowledgements}

None.

\section{Conflict of interest}

The author declares no conflict of interest.

\section{References}

1. Wilkins RL, Stoller JK, Kacmarek RM. Egan's Fundamentals of Respiratory Care. 9th ed. St. Louis, MI: Elsevier; 2009.

2. Archer SL, Weir EK, Wilkins MR. Circulation. 2010;121:2045-2066. 
3. Des Jardins T. Cardiopulmonary Anatomy and Physiology Essentials of Respiratory Care. 5th ed. Clifton Park, NY: Delmar Cengage Learning; 2008.

4. Kamangar N. Secondary pulmonary hypertension treatment and management. 2014.

5. Hill NS, Roberts KR, Preston IR. Postoperative pulmonary hypertension: Etiology and treatment of a dangerous complication. Respiratory Care. 2009;54(7):958-968.

6. ICU Resus Committee. Prolonged Ventilator Weaning Protocol, Policies and Procedures, Southlake Regional Health Centre; 2013
7. Mayo Clinic Staff. Meningioma; 2014.

8. Mayo Clinic Staff. Blood Urea Nitrogen (BUN) Test; 2013.

9. Van Meerbeeck JP, Damhuis RAM, Vos de Wael ML. High postoperative risk after neumonectomy in elderly patients with right-sided lung cancer. European Respiratory Journal. 2002;19(1):141-145.

10. Pharmacy Department, Respiratory Therapy Department, Cardiovascular Surgery. Inhaled Epoprostenol (Flolan), Policies and Procedures, Southlake Regional Health Centre; 2013.

11. Zepeda-Orozco D, Quigley R. Dialysis Disequilibrium Syndrome. Pediatric Nephrology. 2012;27(12):2205-2211. 